\title{
Exploring Google Reverse Image Search to Detect Visual Plagiarism in Interior Design
}

\author{
Abeer A. Alawad \\ King Abdulaziz University
}

This study aims to explore the ability of Google Reverse Image Search (RIS) to detect plagiarism in images in the interior design field. Several image modifications were introduced by retaining the basic concept of the original image. These changes were classified into three categories as follow: a change in the design elements, introduced random changes by adding different objects to the existing image contents, and introduced various image effects. findings show that Google RIS does not take long to find newly uploaded images. Although it cannot detect changes related to the image contents, it can detect changes related to image size and contrast. Overall percentage of the modified images that were detected as matching the original image was only 5\%. By contrast, the net percentage of images retrieved by Google RIS with contents actually related to the uploaded original image was $58.5 \%$. Therefore, Google RIS is inaccurate in detecting any changes in the image contents irrespective of their simplicity, which implies that it cannot help in detection of visual plagiarism.

Keywords: visual plagiarism, image detection, google images, reverse image search, interior design

\section{INTRODUCTION}

Despite the clear advantages of the Internet in terms of accessibility to diverse sources of information, it has contributed to the spread of certain phenomena that negatively affect the lives of individuals as well as society in general. Plagiarism is one such phenomenon that plagues the academic community. Plagiarism is the practice of presenting another person's work, be it their words or ideas, as one's own without giving due credit or mention to the actual author or creator (Bayudan, et al., 2018; Akshay, et al., 2019). However, this phenomenon is not only limited to texts and ideas, but it also extends to images, flow diagrams, and architectural diagrams (Akshay, et al., 2019). While several software programs can detect plagiarism in text, such as iThenticate and Turnitin, programs that can detect plagiarism in images are still in their nascent stages owing to the complexities in the underlying process, as pointed out by Economou (2011).

Plagiarism can be of various forms, such as direct plagiarism (i.e., of another person's work) or selfplagiarism of textual or visual contents (Alawad, et al., 2020). While there is an ongoing debate among researchers regarding the limits of plagiarism, some researchers have proposed a general framework to determine these limits, which may be a complete copy or slight modifications of the original work (Alawad, et al., 2020; Kuruvila, et al., 2017). Nevertheless, even minor modifications need to be properly quantified, for example, in terms of the percentage of plagiarism. However, the lack of a clear referencing system makes the boundary between acceptable and unacceptable practices in visual plagiarism more ambiguous than in text plagiarism, as explained by Economou (Economou, 2011). Moreover, there is a fundamental 
difference in perception of what constitutes a violation of academic integrity between text-based disciplines, such as computer programming, and image-based disciplines, such as the visual arts, which makes the problem more complicated (Simon, 2015).

\section{Design and Plagiarism}

Several studies have indicated that visual plagiarism may occur in a number art and design fields, such as interior design (Alawad, et al., 2020), visual arts (Bayudan, et al., 2018; Simon, 2015), photography (Van Heerden, 2014), and graphic design (Noh, et al., 2018). Researchers, in general, agree that plagiarism of any form is unacceptable, as it has a negative impact on both individual projects and on the field of specialization in general owing to the duplication of original work. Moreover, it hinders the goals of academic programs by barring students from learning effectively, which in turn prevents them from achieving their ambitions in the respective fields (Van Heerden, 2014). Most importantly, plagiarism affects the society at large by preventing original ideas from emerging, thus leading to a stagnation in creativity and innovation (Bayudan, et al., 2018; Alawad, et al., 2020). This practice is a clear indication of the plagiarist's inability to produce original work, and a reflection of their complete lack of respect for the original owner of the work as well as the community that is meant to benefit from the work (Bayudan, et al., 2018).

Several reasons have been attributed to the misuse of visual references by students in the field of art and design. These include the students' lack of knowledge and skill in design, shortage of teacher consultations, incorrect decisions in choosing project topics at an early stage of the design project, and poor time management (Noh, et al., 2018). Alawad et al. (2020) provided an academic point of view of selfplagiarism in students, wherein they state that a fear of failure along with a desire to continue to excel drives students to plagiarize their previous successful works. Some studies have also pointed out that students often cannot separate creative inspiration from copying, which again leads to plagiarism (Coorey, 2018). Nevertheless, all studies conducted so far agree that the ease of accessing images via the Internet is the main reason behind visual plagiarism. In addition, we believe that the ease of manipulating images using various image editing software contributes significantly to visual plagiarism.

Senior academicians and researchers should follow proactive strategies to ensure that a student's work is produced by themselves and not plagiarized. This can be done by following up on the various stages of the development of the project, as well as by having regular discussions with the student; thus, one can objectively judge the student's ability to adequately explain the design stages, which also indicates if their work is original (Noh, et al., 2018). Alawad et al. (2020) proposed that following a student's progress through the different stages of the project is likely to enable their mentors to evaluate the student's skill level, which in turn can help limit self-plagiarism. In addition, researchers should rely on their own personal experiences to differentiate original works from duplicates (Noh, et al., 2018). Economou (2011) noted that it is difficult to define visual plagiarism because of unconscious copying tendencies or because the similarity between two works is merely coincidental or completely unintentional. Nevertheless, it is possible to construct theoretical methods to detect visual plagiarism; however, this needs to be done on a case-by-case basis, as it is difficult to ascertain a person's actual intent behind plagiarism (Economou, 2011).

Notwithstanding the importance of visual plagiarism, this area is lacking in terms of creating accurate programs for detection, clarifying the concept, or setting the acceptable limits of plagiarism in each discipline. According to Alawad et al. (2020), owing to this lack of clarity regarding the acceptable limits, it is difficult to discover or even define visual plagiarism in student-led interior design projects. Contrastingly, researchers also believe that certain elements should not be considered as plagiarism

(Alawad, et al., 2020). Therefore, in this context, Kuruvila et al. (2017) indicated the necessity of creating accurate programs with appropriate input data that can help humans recognize questionable features and detect plagiarism. This further highlights the importance of clarifying acceptable limits and detection criteria for visual plagiarism before developing specialized programs. The importance of accurate visual plagiarism detection programs has also been emphasized by Bayudan et al. (2018), as human detection is 
difficult, inaccurate, and time-consuming. Nevertheless, researchers should also be aware of the new findings in their fields to be able to judge the authenticity of a given work.

\section{Software and Plagiarism}

Relying on text plagiarism detection software to detect plagiarism in images is futile. This is because when a text file is scanned by a system, the images in the file are discarded and plagiarism is detected only in the written text (Ovhal, 2015). Although currently there is no software available to detect visual plagiarism in the field of interior design, there are a few reverse image search (RIS) engines, such as Google, Yandex, and Bing. Peguera (2012) explains how Google RIS works as follows: "Google's robots crawl the Web and make small copies of the images they find. These copies, usually referred to as "thumbnails" due to their reduced size, are stored in Google's servers. They are indexed according to many factors, particularly the contextual information provided by the web page where the image was found." Google offers a multi-language environment and allows users to find images by simply uploading them or by supplying the corresponding URLs instead of using text to search. The Google search results yield a similar image to the one supplied by comparing with images in its database. The benefit of this search tool, according to Google Support (2020), is that it helps to identify where the concerned images are being used, and how common it is to find modified versions of them. Moreover, it helps users to know who is using their pictures with or without their permission. The Google search engine is updated regularly by exploring the Internet and adding websites to the Google index. However, the images need to be publicly available, searchable, and must meet the requirements of Google for the search tool to work.

Wan and Chen (2011) have confirmed that Google RIS relies almost entirely on text-based indexing, wherein it reverses the images based on the textual contents, and not the image contents. A study states that this technique is effective, because it quickly collect the relevant images from a huge number of images available on the Internet; however, the end result is not satisfying (Fergus, et al., 2004). Therefore, it is necessary to think about the concept of Content-Based Image Retrieval (CBIR), in which the image recovery is done based on the actual contents of the images as well as various image features, such as color, shape, texture, and multiple points of interest. These features can be compared directly across images on the Internet to reveal similarities or differences with the image under question (Ovhal, 2015).

A study by Van Heerden (2014) explored the Google ${ }^{\mathrm{TM}}$ Search by Image system to detect visual plagiarism in digital photographs using the color, texture, and shapes therein. This study collected random image samples from the Internet and categorized them into three groups, namely, objects, people, and places. The first group was the control group; the second group was, used to evaluate the accuracy of Google SBI in extracting color and texture from images with modified desaturation, contrast, and hue shift; and the third group was used to evaluate Google's accuracy in extracting shapes from modified image composites. The results of this study showed that the average image retrieval rate differed depending upon whether the images belonged to the objects, people, or places category, as well as on the complexity of the image compositions. This study concluded that photography lecturers can use Google SBI to successfully detect visual plagiarism in images unethically downloaded by students from the Internet. Another recent study evaluated the Google RIS system by classifying the images into the following five categories: "Fashion", "Computers", "Home", "Sports", and "Toys" (Bitirim, et al., 2020). Each category further used five concepts to classify the results of 100 images and to judge their relevance. The results that appeared under Computer, Sports, and Toys had no correlation across the five concepts within each classification, which further confirms that Google RIS needs to evolve more to function better as an image searching tool.

There have been several efforts to detect image similarity either using search engines or using CBIR programs, as proposed by Wan and Chen (Wan, et al., 2011), to improve Google RIS. Some studies have focused on an application for digital artwork that measures the similarities and differences between two images (Bayudan, et al., 2018), while others have explored plagiarism detecting systems in flowcharts (Kuruvila, et al., 2017; Ovhal, 2015). Kalaiarasi et al. (2020) suggested a method for identifying duplicate images that are close to the inquiry image (i.e., having only minor differences) in image search engines to facilitate smooth browsing and avoid displaying multiple copies of the same image during the search. 
Another study proposed a method to speed up the overall image search process (Hellinger, 2020). However, all these methodologies are still being developed.

Note that the methods and search systems that detect plagiarism are still in their early stages of development and experimentation. Furthermore, relying on humans to detect plagiarism in images requires time, effort, and experience, and the results may not be accurate. Until the development of accurate plagiarism detection programs in images, the present study aims to explore the ability of the Google RIS to detect plagiarism in images in the field of interior design. We aim to answer the following questions:

1. Is the Google search engine able to detect similar images recently uploaded to the Internet?

2. Is the search engine able to detect the different kinds of changes related to the image contents?

3. Is the search engine able to detect the changes related to editing effects and image modifications?

4. How relevant are the first ten images returned by the search engine as being related to the original image in the present study?

\section{METHODS}

We evaluated Google RIS by using numerous images, in the field of interior design, containing several differences from the original image in question. The present study was conducted in the academic year 2020 .

\section{Procedure}

We followed a procedure composed of several stages. The outline of the pre-testing stage is as follows:

- Websites, such as Pinterest and Twitter, that are detected by Google RIS, were first identified by testing a random image.

- Random images from the author's account on Twitter, from a month-old to a year-old, were examined to ensure that the author's website appeared in the search engine. These images appeared in the search result as "pages that contain matching images".

- A Google RIS was conducted to verify that it returns images, sketches, or technical drawings identical to the original image.

- A drawing of a dining room was created by the author using Autodesk Revit 2020 and Lumion 10.0.1, as shown in Fig. 1, which was used as the original image. The reason for choosing an original drawing, and not any random image available on the Internet, was to ensure the integrity of the image, or, in other words, free from any factors that may make it too easy to obtain or conceal. Such an image also makes it easy to introduce changes within it The drawing was edited using Adobe photoshop.

- The original image was then uploaded to the author's personal accounts on different wellknown platforms, such as Instagram, Pinterest, Twitter, and ResearchGate. After sufficient time had passed to ensure that the image appeared in the Google RIS, a search was conducted for the image every day since the first upload to estimate the time it took for it to appear in the search. Note that the image was searched at different times and from different devices to ensure that it is not linked to any information connected to the author's accounts.

The changes introduced in the original image were classified into three categories, as shown in Table 1. A total of 40 changes were made, and a code was assigned to each change, as follows:

- First category: In this category, 30 out of the 40 images have changes in the design elements of the original image, namely: lines, shape, size, texture; color, and mass and space. There is a total of five images under each of these six elements, which are categorized as follows: three of the images contain a unique change each; one image contains two changes; the final image contains three changes.

- Second category: In this category, three images were created by randomly changing the image contents either by adding new objects or by manipulating existing objects. 
- Third category: In this category, seven images were created based on the following image effects (one change per image only): digital sketch effects, digital watercolor effects, desaturation (black and white), contrast increase by +50 , hue shift by $-180^{\circ}$, partial image consideration, and change in size. Note that some of these changes are similar to those in the study by Van Heerden (Van Heerden, 2014), except for the desaturation, contrast increase, and hue shift, as well as the image contents and total number of images studied.

\section{FIGURE 1}

ORIGINAL IMAGE OF A DINING ROOM

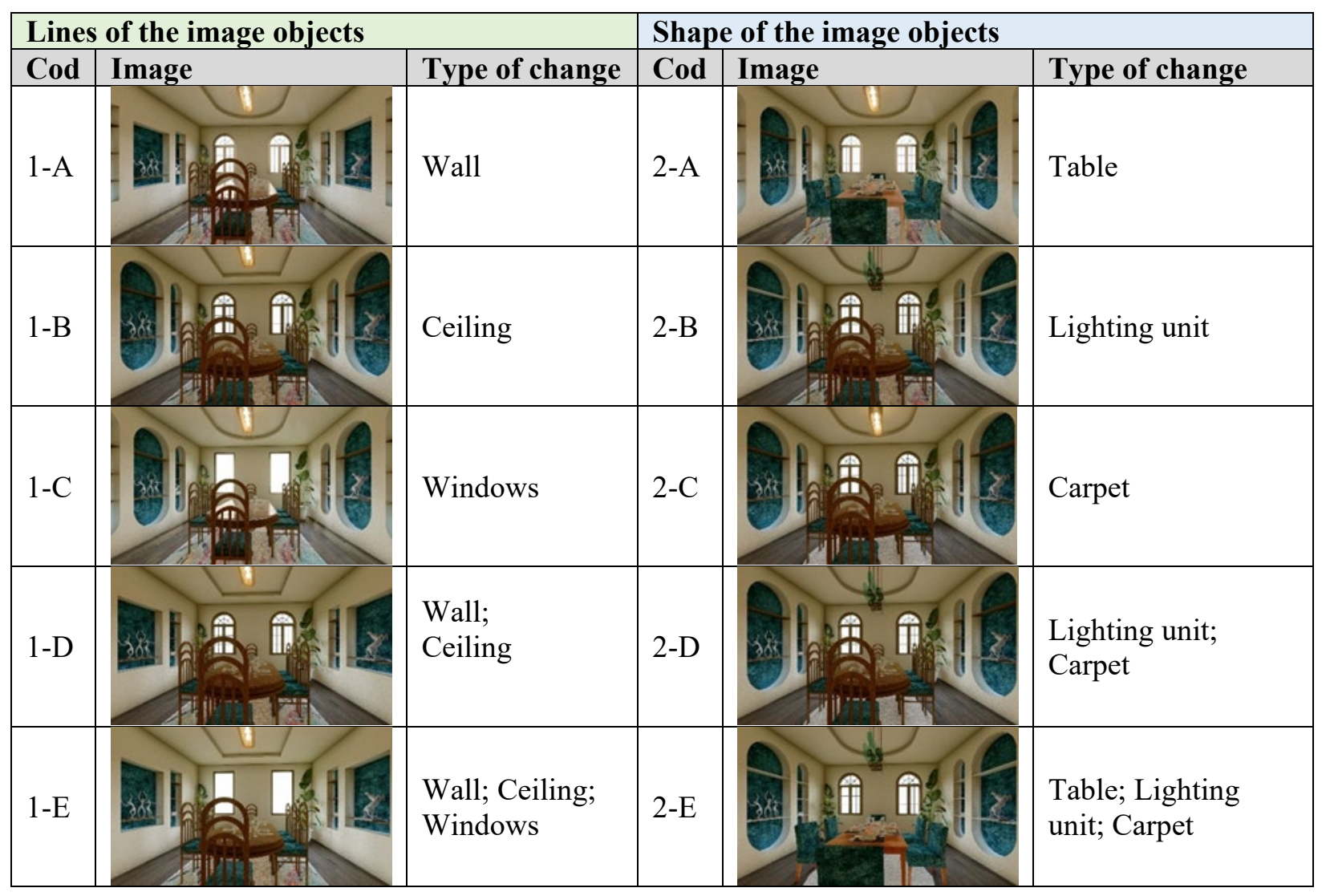




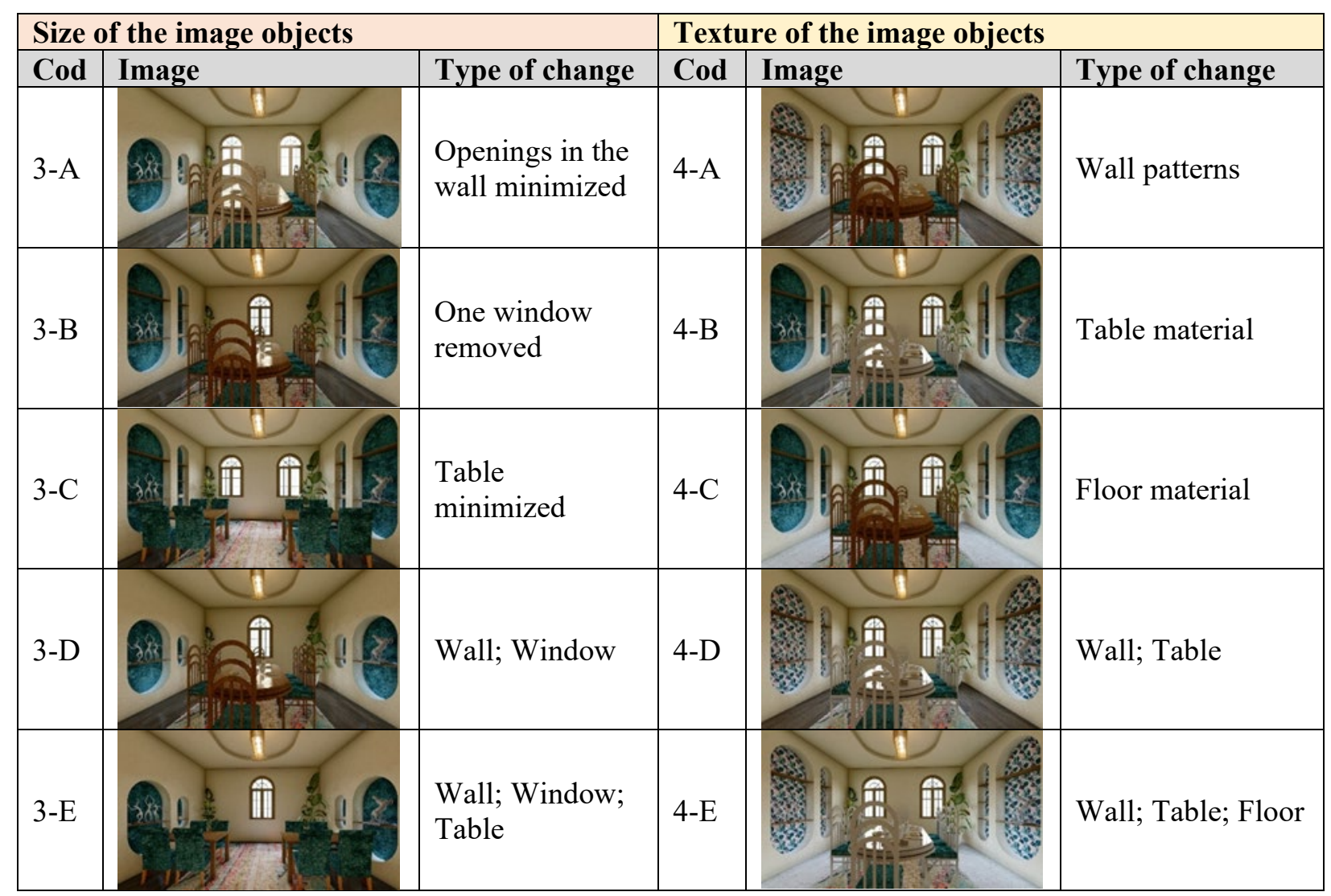

\begin{tabular}{|l|l|l|l|l|l|l|}
\hline Color of the image objects & \multicolumn{3}{l|}{ Mass and space of the image objects } \\
\hline Cod & Image & Type of change & Cod & Image & Type of change \\
\hline 5-A & Achromatic & 6-A & & Walls made \\
prominent
\end{tabular}


TABLE 1

CATEGORIES OF CHANGES MADE TO THE ORIGINAL IMAGE

\begin{tabular}{|l|l|l|l|}
\hline \multicolumn{3}{|l|}{ Random changes in the image objects } & Type of change \\
\hline Cod & Image & Size, location, and number of windows changed; \\
\hline $7-A$ & & & \\
\hline
\end{tabular}

Note that, as shown in Table 1, none of the 40 changes affect the basic concept of the original image, which is a dining room; thus, simple changes were considered to explore the accuracy of Google RIS system in tracking images and set the expectations of our investigation. In the present study, we checked all the images that appeared in our search but considered only the top ten images "as being detected" because they were identified as the closest to the original image by the system.

\section{RESULTS}

The image appeared in Google RIS one week after it was uploaded under "pages that contain matching images," which corresponded to the author's Twitter account only, although it was uploaded to other websites at the same time. Table 2 shows the results of detection of all 40 images and the corresponding keywords suggested by Google RIS.

The results presented in Table 2 show that Google classified the images with different keywords even though all the changes made adhered to the basic concept of a dining room. Moreover, the keywords assigned did not appear to consider the exact nature of the change introduced in the image. Moreover, completely different changes were often assigned the same keywords. For example, none of the five images having changes in the line elements of the objects (i.e., category 1) were detected as matching the original image. Images 1-A to 1-D were assigned the keyword "furniture style", whereas image 1-E was assigned "daylight" even though it contained three different changes in the lines of the wall, ceiling, and window (see Table 1). Similar interpretations can be drawn about the images in categories 2-7. Interesting results were obtained only when editing effects were introduced in the images, namely in category 8 . The images with code $8-\mathrm{D}$, whose contrast was increased by +50 , and code $8-\mathrm{G}$, whose size was decreased, were detected to be visually matching the original image. 
TABLE 2

IMAGE DETECTION RESULTS AND CORRESPONDING GOOGLE RIS KEYWORDS

\begin{tabular}{|c|c|c|c|c|}
\hline $\begin{array}{l}\text { Image } \\
\text { code }\end{array}$ & Suggested Keyword & Detection & Website & Notes \\
\hline 0 & Furniture style & $\checkmark$ & Twitter & $\begin{array}{l}\text { Matching images from } \\
\text { author's account }\end{array}$ \\
\hline $1-\mathrm{A}$ & Furniture style & $x$ & - & $\begin{array}{l}\text { No matching images } \\
\text { found }\end{array}$ \\
\hline $1-B$ & Furniture style & $x$ & - & \\
\hline $1-\mathrm{C}$ & Furniture style & $x$ & & \\
\hline $1-\mathrm{D}$ & Furniture style & $x$ & - & \\
\hline $1-\mathrm{E}$ & Daylight & $x$ & - & \\
\hline $2-\mathrm{A}$ & Kitchen \& dining room table & $x$ & - & \\
\hline $2-\mathrm{B}$ & Furniture style & $x$ & - & - \\
\hline $2-\mathrm{C}$ & Furniture style & $x$ & - & - \\
\hline 2-D & Furniture style & $x$ & - & - \\
\hline 2-E & Daylight & $x$ & - & - \\
\hline $3-\mathrm{A}$ & Daylight & $x$ & - & - \\
\hline $3-\mathrm{B}$ & Daylight & $x$ & - & - \\
\hline $3-\mathrm{C}$ & Daylight & $x$ & - & - \\
\hline $3-\mathrm{D}$ & Daylight & $x$ & - & - \\
\hline $3-\mathrm{E}$ & Daylight & $x$ & - & - \\
\hline $4-\mathrm{A}$ & Furniture style & $x$ & - & - \\
\hline $4-B$ & Daylight & $x$ & - & - \\
\hline $4-\mathrm{C}$ & Furniture style & $x$ & - & - \\
\hline $4-\mathrm{D}$ & Arch & $x$ & - & - \\
\hline $4-\mathrm{E}$ & Arch & $x$ & - & - \\
\hline $5-\mathrm{A}$ & Daylight & $x$ & - & - \\
\hline $5-\mathrm{B}$ & Furniture style & $x$ & - & - \\
\hline $5-\mathrm{C}$ & Furniture style & $x$ & - & - \\
\hline $5-\mathrm{D}$ & Daylight & $x$ & - & - \\
\hline $5-\mathrm{E}$ & Kitchen \& dining room table & $x$ & - & - \\
\hline $6-\mathrm{A}$ & Daylight & $x$ & - & - \\
\hline $6-\mathrm{B}$ & Furniture style & $x$ & - & - \\
\hline $6-\mathrm{C}$ & Kitchen \& dining room table & $x$ & - & - \\
\hline $6-\mathrm{D}$ & Daylight & $x$ & - & - \\
\hline $6-\mathrm{E}$ & Furniture style & $x$ & - & - \\
\hline 7-A & Furniture style & $x$ & - & - \\
\hline 7-B & Dog & $x$ & - & - \\
\hline $7-\mathrm{C}$ & Daylight & $x$ & - & - \\
\hline 8-A & Daylight & $x$ & - & All sketches \\
\hline $8-\mathrm{B}$ & Daylight & $x$ & - & \\
\hline $8-\mathrm{C}$ & Kitchen \& dining room table & $x$ & - & - \\
\hline 8-D & Furniture style & $\checkmark$ & Twitter & $\begin{array}{l}\text { Matching images from } \\
\text { author's account }\end{array}$ \\
\hline $8-\mathrm{E}$ & Daylight & $x$ & - & 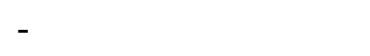 \\
\hline $8-\mathrm{F}$ & Mission style furniture & $x$ & - & - \\
\hline $8-\mathrm{G}$ & Furniture style & $\checkmark$ & Twitter & $\begin{array}{l}\text { Matching images from } \\
\text { author's account }\end{array}$ \\
\hline
\end{tabular}


In Table 3 we summarize our image detection results. We observe that changes introduced in the image contents resulted in $0 \%$ matching or visually similar images, whereas editing effects led to a $2 \%$ detection rate. The overall percentage of images that were detected as matching the original image in this study was $5 \%$.

To detect the accuracy of the Google search engine, we analyzed the first ten images that were selected by Google as being related to the original image of the dining room (under each category of change). However, these results were different from those presented in Table 3. In Table 4, we present the number and percentage of images returned by Google, whose contents was related to the original image under each category of change introduced in this work. We observe that $58.5 \%$ of the total number of images detected by Google was classified as being related to the original image, all adhering to the same concept of a dining room.

TABLE 3

NUMBER AND PERCENTAGE OF IMAGES DETECTED IN ALL CATEGORIES

\begin{tabular}{|l|l|l|l|}
\hline Category & Number of Changes & Number of Detections & Percentage Detection (\%) \\
\hline Lines & 5 & 0 & 0 \\
\hline Shape & 5 & 0 & 0 \\
\hline Size & 5 & 0 & 0 \\
\hline Texture & 5 & 0 & 0 \\
\hline Color & 5 & 0 & 0 \\
\hline Mass and space & 5 & 0 & 0 \\
\hline Random changes & 3 & 0 & 0 \\
\hline Editing effects & 7 & 2 & 28.6 \\
\hline Total & 40 & 2 & 5.0 \\
\hline
\end{tabular}

TABLE 4

NUMBER AND PERCENTAGE OF GOOGLE DETECTED IMAGES WITH RELATED CONTENT IN ALL CATEGORIES

\begin{tabular}{|l|l|l|l|}
\hline Category & Number of Changes & Number of Related Images & $\begin{array}{l}\text { Percentage } \\
\text { Detection (\%) }\end{array}$ \\
\hline Lines & 5 & 49 & 98.0 \\
\hline Shape & 5 & 42 & 84.0 \\
\hline Size & 5 & 38 & 76.0 \\
\hline Texture & 5 & 21 & 70.0 \\
\hline Color & 5 & 26 & 52.0 \\
\hline Mass and space & 5 & 24 & 48.0 \\
\hline Random changes & 3 & 33 & 47.1 \\
\hline Editing effects & 7 & 1 & 2.0 \\
\hline Total & 40 & 234 & 58.5 \\
\hline
\end{tabular}

\section{DISCUSSION}

Google RIS is free, easy-to-use, multilingual and has several advantages that are absent in other websites. It can be used for various image related purposes; however, the aim of the present study was to explore the ability of Google RIS to detect plagiarism in images in the field of interior design. To this end, several questions were prepared. Our results show that the original uploaded image took considerable time to appear in Google RIS. In fact, Google RIS did not detect all the websites in which the image was uploaded. In addition, Google classified each image with a different keyword even though the changes 
introduced in the images did not affect the basic concept of the image, which in our case was a dining room. Interestingly, Google RIS was not able to find visual similarities with the original image for most of the categories of changes introduced (i.e., for image codes 1-A to 8-C, 8-E and 8-F). Although the changes in the images included contents as well as various editing effects, Google failed to detect any similarities with the original image. This implies that Google RIS does not read the contents of the images, which agrees with the findings of previous studies that the search is almost exclusively text-based and not based on contents (Wan, et al., 2011; Ovhal, 2015). Google RIS could successfully identify only the images 8-D, whose contrast was increased by +50 , and $8-G$, whose hue was shifted by $-180^{\circ}$, which agree with the results in (Van Heerden, 2017). This indicates that Google search is inaccurate in detecting any changes in the image contents no matter how simple it is, except in these two cases; thus, it cannot facilitate the detection of visual plagiarism in the field of interior design.

Note that in the present study, new images were created and uploaded on the Internet; however, the detection results were different when existing images were chosen from the Internet, downloaded, manipulated and uploaded again. In addition, the analysis was made on numerous images, and the appearance was uneven, which may be affected by the site on it, or the word accompanying the image, or even the input on the image. Thus, a positive characteristic of Google RIS is that it successfully reveals images that are unprocessed or have been recently uploaded to the Internet. Our results also indicate that the Google-suggested keyword for a modified image is not related to the kind or number of changes in the image. Moreover, we found that Google RIS classified about half of the images found on the internet, based on the first ten search results, as matching the original image.

\section{CONCLUSION}

Only a few studies on visual plagiarism have been conducted in the field of art and design; however, to the best of our knowledge, no such study exists in the field of interior design. This may be due to the complexity of the design process that requires extremely accurate software, which has been carefully programmed with the knowledge of what constitutes an unacceptable or an acceptable duplicate image. It is clear that organizations or programmers will not be able to create suitable visual plagiarism detection applications unless interior design specialists collaborate with them to clarify the acceptable limits. In the absence of a program, the situation will remain dependent on humans, for whom it is a fundamentally difficult task, as it is next to impossible to keep pace with the amount of information being uploaded to the Internet on a regular basis. We also note that existing programs are incapable of understanding the depth of an image and its components, such that minor changes in the image may go unnoticed. Although changes introduced by certain editing effects may be captured by existing software, it is still quite superficial. However, projects in the field of interior design depend solely on the contents of the images; thus, existing programs are ineffective in detecting visual plagiarism in this field.

There are several topics that deserve future attention in the field of interior design, such as clarifying the acceptable limits of plagiarism and creating a program that can detect similarity in projects to limit plagiarism, which in turn would foster innovation. The guaranteed solution for now is to spread awareness among students about visual plagiarism, and thus endeavor to promote scientific integrity and preserve the copyrights of the original creators.

\section{ACKNOWLEDGEMENT}

The authors would like to thank interior designer Basma Alharbi for helping with the technical drawings in this work. 


\section{REFERENCES}

Akshay, S., Chaitanya, B.N., \& Kumar, R. (2019). Image plagiarism detection using compressed images. IJITEE, 8, 1423-1426.

Alawad, A.A., Bettaieb, D.M., \& Malek, R.B. (2020). Self-plagiarism in students' interior design projects from an academic perspective. International Journal of Management, 11, 1370-1382.

Bayudan, J.H.C., Pati, F., Jr., Slater, R.E., \& Palaoag, T.D. (2018, September 17-18). Safe art: A digital artwork plagiarism detector. In Theory and practice of computation. Proceedings of the Workshop on Computation: Theory and Practice (WCTP 2018) (p.167). Manila, The Philippines: CRC Press.

Bitirim, Y., Bitirim, S., Ertugrul, D.C., \& Toygar, O. (2020). An evaluation of reverse image search performance of Google. In IEEE 44th Annual Computers, Software, and Applications Conference (COMPSAC) (pp. 1368-72). IEEE.

Coorey, J. (2018). Removing plagiarism from the design process: Stimulating creativity and originality in the design classroom. International Journal of Design Education, 12(1), 11-19. doi:10.18848/2325-128X/CGP/v12i01/11-19

Economou, I. (2011, September 7-8). The problem with plagiarism. In Proceedings of the Sixth International DEFSA Conference: 20/20 Design Vision, University of Johannesburg (pp. 79-86).

Fergus, R., Perona, P., \& Zisserman, A. (2004). A visual category filter for Google Images. In Lecture Notes in Computer Science European Conference on Computer Vision (pp. 242-256). Berlin, Heidelberg. Berlin: Springer. doi:10.1007/978-3-540-24670-1_19

Google Support 2020. (n.d.). Retrieved from https://support.google.com/websearch/answer/112511?hl=en\&ref_topic=3180360\#

Hellinger correlation coefficient. (2020). Int. J. Adv. Res Eng. Technol., 11 , 846-859.

Hurtik, P., \& Ftip, H.P. (2015). A tool for an image plagiarism detection. In 7th International Conference of Soft Computing and Pattern Recognition (SoCPaR) (pp. 42-47). IEEE Publications.

Kalaiarasi, G., Maheswari, M., Devadas, P., \& Selvi, M. (2020). Detecting near duplicate images using. International Journal of Advanced Research in Engineering and Technology, 11(6), 846-859. doi: 10.34218/IJARET.11.6.2020.076

Kuruvila, J.S., Midhun Lal, V.L., Roy, R., Baby, T., Jamal, S., \& Sherly, K.K. (2017). Flowchart plagiarism detection system: an image processing approach. Procedia Comput. Sci., 115, 533-40.

Noh, M.A.M., Nudin, A.L.A., Abdullah, N.N.N., Shamsudin, W.N.K., \& Harun, M F. (2018). Plagiarism in graphic design. In Proceedings of the Art and Design International Conference (AnDIC 2016) Singapore (pp. 391-400). Berlin: Springer.

Ovhal, P. (2015). Detecting plagiarism in images. In International Conference on Information Processing (ICIP) (pp. 85-89). Pune, India: IEEE Publications.

Peguera, M. (2012). Copyright issues regarding Google Images and Google cache. In Google and the law (pp. 169-202). T M C Asser Press.

Simon, S. (2015). Academic integrity in non-text based disciplines. In T. Bretag (Ed.), Handbook of academic integrity (pp. 763-782). Singapore: Springer.

Van Heerden, L. (2014). Detecting internet visual plagiarism in higher education photography with Google ${ }^{T M}$ search by image: Proposed upload methods and system evaluation [Doctoral Dissertation]. Bloemfontein, South Africa: Central University of Technology: Free State.

Wan, Y., Liu, X., \& Chen, Y. (2011). Online image classifier learning for Google Image search improvement. In IEEE International Conference on Information and Automation (pp. 103-110). IEEE Publications. 